\title{
Macrophage-derived Apoptotic Vesicles Regulate Fate Commitment of Mesenchymal Stem Cells via miR155
}

\section{Yuan Zhu}

Peking University School of Stomatology

Xiao Zhang

Peking University School of Stomatology

\section{Kunkun Yang}

Peking University School of Stomatology

\section{Yuzi Shao}

Peking University School of Stomatology

\section{Ranli Gu}

Peking University School of Stomatology

Xuenan Liu

Peking University School of Stomatology

Hao Liu

Peking University School of Stomatology

Yunsong Liu ( $\square$ liuyunsong@hsc.pku.edu.cn )

Peking University School of Stomatology https://orcid.org/0000-0001-8364-1898

\section{Yongsheng Zhou}

Peking University School of Stomatology

\section{Research Article}

Keywords: Macrophage, apoptotic vesicles, adipogenesis, osteogenesis, MSCs, microRNA155

Posted Date: November 29th, 2021

DOI: https://doi.org/10.21203/rs.3.rs-1101843/v1

License: (c) (1) This work is licensed under a Creative Commons Attribution 4.0 International License. Read Full License 


\section{Abstract \\ Background}

In tissue engineering, mesenchymal stem cells (MSCs) are common seed cells because of abundant sources, strong proliferation ability and immunomodulatory function. Numerous researches have demonstrated that MSC-macrophage crosstalk played a key role in the tissue engineering. Macrophages could regulate the differentiation of MSCs via different molecular mechanisms, including extracellular vesicles. Apoptotic macrophages could generate large amounts of apoptotic vesicles (apoVs), whereas the functions of macrophage-derived apoVs remain largely unknown. There was no research to clarify the role of macrophage-derived apoVs in MSC fate choices. In this study, we aimed to characterize macrophage-derived apoVs, and investigate the roles of macrophage-derived apoVs in the fate commitment of MSCs.

\section{Methods}

We characterized macrophage-derived apoVs, and investigated their role in MSC osteogenesis and adipogenesis in vitro and in vivo. Furthermore, we performed microRNA loss- and gain- of function experiments and western blot to determine the molecular mechanism.

\section{Results}

We found that macrophage-derived apoVs inhibited osteogenesis and promoted adipogenesis in vitro and in vivo. In mechanism, apoVs regulated osteogenesis and adipogenesis of MSCs by delivering microRNA155 (miR155).

\section{Conclusions}

Macrophage-derived apoVs could regulate the osteogenesis and adipogenesis of MSCs through delivering miR155, which provided novel insights for MSC-mediated tissue engineering.

\section{Background}

Over the past several decades, tissue engineering has been an important research direction to repair defects in the regenerative medicine(1-5). It involves three crucial components: seed cells, scaffolds, and bioactive molecules(6-8). Seed cells should have self-renewal ability and differentiation potential, be able to establish stable cell lines, and have the lowest possible antigenicity(9-11). MSCs have been widely applied in regenerative medicine research because of their abundance, strong proliferation ability and immunomodulatory function(12-16). 
Macrophages are important modulators of host defense. Under physiological or pathological conditions, resting macrophages (M0) can polarize into proinflammatory phenotype (M1) and anti-inflammatory phenotype (M2)(17-20). Many investigations have indicated that MSC-macrophage crosstalk occupied an important role in the osteogenic differentiation of MSCs through different molecular mechanisms, especially through extracellular vehicles (EVs)(21-23). Kang M et al. reported that M0 and M2 EVs could promote bone regeneration, while M1 EVs could inhibit bone regeneration(21). Xiong $Y$ et al. showed that exosomes secreted by M2 macrophages promoted osteogenesis, whereas exosomes secreted by M1 macrophages inhibited osteogenesis of bone mesenchymal stem cells(22). Exosomes derived from M1 macrophages significantly promoted the expression of PPAR- $y$ and lipid droplet formation(23). Therefore, vesicles produced by macrophages in different polarization states have different effects on MSCs.

Macrophages can secrete vesicles in physiological state, they can also secrete a large number of apoptotic vesicles (apoVs) under apoptotic state, but little is known about the macrophage-derived apoVs. Apoptosis is the spontaneous and orderly death of cells and contributes to maintenance of homeostasis(24-26). ApoVs are rich in protein, RNAs and lipids, and they are a key intercellular communication medium (27). Our previous study has investigated that functional efferocytosis of apoVs could restore liver macrophage homeostasis and ameliorates type 2 diabetes(28). In 2018, Liu D et al. reported the apoptotic bodies could maintain bone homeostasis in physiological and pathological conditions and may have therapeutic potential for osteoporosis(29). In 2020, Liu H et al. showed that extensive apoptosis occurred soon after stem cell transplantation, and a large number of apoptotic bodies were released, enhancing angiogenesis and having a therapeutic effect(30). However, the regulation of macrophage-derived apoVs on fate choices of MSCs is unclear. In this study, we detected that macrophage-derived apoVs could promote adipogenesis and inhibit osteogenesis of MSCs both in vitro and in vivo, implicating miR155 in the mechanism by which apoVs regulate MSC adipogenesis and osteogenesis. Our study not only clarified the novel functions of macrophage-derived apoptotic vesicles on differentiation of MSCs, but also gave hints for tissue engineering.

\section{Methods}

\section{Culture of RAW 264.7 macrophages and MSCs.}

Primary RAW264.7 macrophages and hASCs were obtained from ScienCell Research Laboratories and these cells were grown in proliferation medium (PM) which contained $10 \%(v / v)$ fetal bovine serum (FBS) and antibiotics in DMEM.

\section{Osteogenic and adipogenic induction of MSCs.}

The osteogenic medium (OM) contained $10 \mathrm{nM}$ dexamethasone, $200 \mu \mathrm{M}$ ascorbic acid and $10 \mathrm{mM} \beta$ glycerophosphate in DMEM with $10 \%(\mathrm{v} / \mathrm{v})$ FBS and antibiotics. The adipogenic medium (AM) contained $100 \mathrm{nM}$ dexamethasone, $50 \mathrm{nM}$ insulin, $0.5 \mathrm{mM}$ 3-isobutyl-1-methylxanthine and $200 \mu \mathrm{M}$ indomecin in DMEM with $10 \%(v / v)$ FBS and antibiotics. 


\section{TUNEL staining.}

Apoptosis was detected using the TUNEL Cell Apoptosis Detection Kit (Applygen). Briefly, normal or apoptotic macrophages were fixed and treated with $0.2 \%$ Triton X-100. Images were obtained using a fluorescence microscope (Olympus) after exposure to rhodamine fluorescein (red) labeled dUTP solution in the dark for $1 \mathrm{~h}$.

\section{Isolation and purification of apoVs.}

ApoVs were collected as described previously with modifications(28). When macrophages reached 80$90 \%$ confluence, serum-free medium containing $500 \mathrm{nM}$ staurosporine (STS) (Enzo Life Sciences) was replaced and the supernatant was collected $12 \mathrm{~h}$ later. The supernatant was centrifuged at $800 \mathrm{~g}$ for 10 min and the precipitate was discarded, then, at $16,000 \mathrm{~g}$ for $30 \mathrm{~min}$ and the precipitate was collected and washed twice with filtered PBS. The Pierce BCA Protein Assay Kit (Thermo Scientific) was used to assess apoV concentration.

\section{Identification of apoVs.}

The morphology of the apoVs was observed by transmission electron microscopy (TEM). The apoVs were fixed and dropped onto a carbon-coated copper net. After air drying, apoVs were twice negatively stained with $1 \%$ uranyl acetate. Images were captured at $120 \mathrm{kV}$ using an HT7700 TEM (Hitachi). Particle size of apoVs was determined by nanoparticle tracking analysis (NTA) using a Nano Sight NS300 (Malvern).

\section{ApoV uptake by MSCs.}

ApoVs were labeled with PKH-26 (Sigma-Aldrich) as described previously with modifications(31). The labeled apoVs were washed in filtered PBS at $16,000 \mathrm{~g}$ for $30 \mathrm{~min}$. Next, apoVs were incubated with MSCs for 4 and $8 \mathrm{~h}$. After incubation, the cells were fixed. Then, the cells were treated with $0.1 \%$ TritonX-100 at room temperature for $7 \mathrm{~min}$, washed twice with PBS. $5 \mathrm{ug} / \mathrm{mL}$ FITC-Cyclopeptide (Sigma-Aldrich) was added. A solution of 6-diamidine-2-phenylindole (DAPI) was used to stain nuclei. The images were obtained using an LSM 5 EXCITER (Carl Zeiss).

\section{Oil red 0 staining and quantification.}

The MSCs were cultured in PM, AM, and AM with apoVs (AM + apoVs), respectively. After 14 days, oil red $O$ staining and quantitative experiment were performed. Oil red $O$ working solution was added to stain the cells. The staining was observed under the microscope at any time. For quantitative evaluation, stained cells were added to $100 \%$ isopropyl alcohol and dissolved for $10 \mathrm{~min}$, the absorbance at $500 \mathrm{~nm}$ was measured.

\section{Alkaline phosphatase (ALP) staining and ALP activity, Alizarin red S (ARS) staining and quantification.}


The MSCs were divided into three groups: PM, OM, and OM added apoVs (OM + apoVs). After osteogenic induction for 7 days, ALP staining and activity assays were performed. BCIP / NBT staining kit (Beyotime Biotechnology) was used for ALP staining. ALP activity was quantified using an ALP Assay Kit (Nanjing Jiancheng) and the absorbance at $520 \mathrm{~nm}$ was measured. ALP activity (U/gprot) per gram of protein was calculated.

ARS staining and quantification were performed 14 days after osteogenic induction. Cells were incubated with Alizarin red buffer (Sigma-Aldrich). And $100 \mathrm{nM}$ cetylpyridine solution was added for the quantification. The absorbance at $562 \mathrm{~nm}$ was measured.

\section{Real-time quantitative polymerase chain reaction (RT-qPCR).}

Total RNA was extracted from cells using TRIzol reagent (Invitrogen) and cDNA was synthesized using a reverse transcription system (TaKaRa) RT-qPCR was performed on the ABI Prism 7500 real-time PCR System using SYBR Green Master Mix. GAPDH and U6 were used as the reference genes. The primer sequences were listed in Table 1.

\section{Western blot analysis}

Cells were lysed in RIPA lysis buffer and protein extracts were subjected to 10\% SDS-PAGE and transferred to polyvinylidene difluoride membranes. The membranes were incubated with the primary antibodies overnight and then with a peroxidase-conjugated secondary antibody. The ECL Kit (NCMbio) was used to detect protein bands.

In vivo implantation of MSCs.

The MSCs were cultured in (PM, AM, AM + apoVs) for 7 days, and mixed with the collagen membrane scaffolds. The number of cells inoculated in each tube was $1 \times 10^{6}$, and the volume of the collagen membrane scaffold was about $8 \mathrm{~mm} \times 8 \mathrm{~mm} \times 2 \mathrm{~mm}$. The mixtures ( $\mathrm{n}=10$ per group) were implanted into $\mathrm{BALB} / \mathrm{c}$ nude mice. The tissues were harvested after 6 weeks. All samples were performed by hematoxylin and eosin (H\&E) staining and oil red $O$ staining.

The MSCs were cultured in (PM, PM + apoVs), and mixed with $\beta$-TCP (RB-SK-005 G) (about $3 \mathrm{~mm} \times 2 \mathrm{~mm}$ $\times 2 \mathrm{~mm}$ ), and the number of cells inoculated in each tube was $1 \times 10^{6}$. The tissues were harvested after 8 weeks. All samples were performed by H\&E staining and Masson trichrome staining.

\section{MiRNA transient infection.}

Mimics-miR155 (miR155), mimics-negative control (miR-NC), inhibitor-miR155 (inhi-miR155) and inhibitor-negative control (inhi-NC) were purchased from Sangon Biotech. The sequences were listed in Table 2. MiRNAs were transfected using Lipofectamine 3000 (Invitrogen) onto macrophages were cultured at $60-70 \%$ confluence. After 48 h, cells were collected for RNA analysis. ApoVs were obtained as described above. 


\section{Statistical analysis.}

SPSS 19.0 software was used for statistical analysis. Comparisons of two groups were performed using independent two-tailed Student's t tests, and comparisons of more than two groups by one-way ANOVA and Tukey's post hoc test. Data were expressed as mean \pm standard deviation. A value of $P<0.05$ was considered indicative of statistical significance.

\section{Results}

\section{Characterization of macrophage-derived apoVs and apoV uptake by MSCs.}

STS was used to induce apoptosis of macrophages. Apoptotic and normal macrophages were observed under fluorescence microscope. A larger number of TUNEL positive stained cells (red) were observed in the STS group, while control group had seldom stained cells (Fig. 1A). Apoptotic macrophages could generate lots of apoVs, the output of apoVs was much higher than exosomes (Fig.S1). TEM showed that apoVs had a cup-shaped morphology and the diameter was about $200 \mathrm{~nm}$ (Fig. 1B). Nanoparticle tracking analysis showed that the diameter distribution of apoVs was $240.6 \pm 115 \mathrm{~nm}$ (Fig. 1C). To investigate whether apoVs could be ingested by MSCs, MSCs were cultured with PKH-26-labeled apoVs (red) for $4 \mathrm{~h}$ and $8 \mathrm{~h}$, respectively. The nuclei of MSCs were stained with DAPI (blue). The F-actin of MSCs was stained with phalloidin (green). Confocal laser microscopy showed that red stained particles appeared around the MSCs nucleus after 4 hours, and the number of red stained particles increased after 8 hours (Fig. 1D).

\section{Macrophage-derived apoVs promoted adipogenesis of MSCs in vitro.}

We set up different concentration gradients $(0.5,1,2,4 \mu \mathrm{g} / \mathrm{mL})$ to clarify the optimal concentration of apoVs, and cultured MSCs in OM and AM respectively for 7 days. In AM culture, $2 \mu \mathrm{g} / \mathrm{ml}$ apoVs significantly increased the PPARY expression; in OM culture, 2 and $4 \mu \mathrm{g} / \mathrm{ml}$ apoVs inhibited the RUNX2 expression levels more significantly (Fig.S2). Therefore, we used $2 \mu \mathrm{g} / \mathrm{mL}$ apoVs in the subsequent experiments. Next, to further investigate the role of macrophage-derived apoVs in MSC adipogenesis, we treated MSCs under AM with or without apoVs. After 14 days, the cells were stained with oil red 0 . Fig. $2 \mathrm{~A}$ showed a great number of red-stained lipid droplets were formed when cells were cultured in AM, while the lipid droplets were significantly increased in MSCs treated with apoVs. In AM, oil red 0 quantitative analysis (Fig. 2B) of the group under stimulation by apoVs was higher than group without apoVs $(P<$ 0.05). In addition, $P P A R Y$ and $C / E B P a$ expression levels of the group treated with apoVs were significantly higher than group without apoVs $(P<0.001)$ (Fig. 2C). Moreover, the protein expression of PPARy was upregulated during adipogenesis in the group treated with apoVs compared with the group without apoVs (Fig. 2D, 2E).

\section{Macrophage-derived apoVs inhibited osteogenesis of MSCs in vitro.}


To further explore the effect of macrophage-derived apoVs on osteogenesis of MSCs, we treated MSCs under OM with or without apoVs for 7 days, the cells were examined by ALP staining (Fig. 3A) and ALP quantification (Fig. 3B), the results showed that apoVs significantly inhibited osteogenic differentiation of MSCs. The expression of $A L P$ and RUNX2 was significantly decreased by apoVs (Fig. 3C). After treated MSCs under OM with or without apoVs for 14 days, the ARS staining and quantification showed similar results that apoVs could inhibit osteogenic differentiation of MSCs (Fig. 3D, 3E). Moreover, RUNX2 and $B G L A P$ expression was significantly decreased by treatment with apoVs for 14 days in OM (Fig. 3F). In addition, the protein expression of RUNX2 was down-regulated during osteogenesis by apoVs (Fig. 3G, $3 \mathrm{H})$.

\section{Macrophage-derived apoVs promoted adipogenesis of MSCs in vivo.}

To examine the role of macrophage-derived apoVs in MSC adipogenic differentiation in vivo, we combined MSCs (PM, AM, and AM + apoVs) with collagen sponges and implanted them into nude mice. H\&E staining showed that the AM + apoVs group had more adipose tissue-like structures than the AM group, whereas the PM group showed a large amount of collagen membrane scaffold and no adipose tissue-like structures (Fig. 4A). Oil red $O$ staining showed that the $A M+$ apoVs group had more red-stained adipose tissue-like structures than the AM group (Fig. 4B). Therefore, macrophage-derived apoVs could promote adipogenesis of MSCs in vivo.

\section{Macrophage-derived apoVs inhibited osteogenesis of MSCs in vivo.}

In order to determine the role of macrophage-derived apoVs in MSC osteogenesis in vivo, we mixed groups (PM and PM + apoVs) with $\beta$-TCP and cell-free $\beta$-TCP, then implanted them into nude mice. H\&E staining (Fig. 5A) showed that the PM group had more new, strongly eosinophilic tissue compared to the $\beta$-TCP group, whereas the PM + apoVs group had less bone tissue-like structures than PM group. Masson staining (Fig. 5B) showed more blue-green collagen fibers in the PM group than the $\beta$-TCP group, whereas the PM + apoVs group had less blue-green collagen fibers than the PM group. Therefore, macrophagederived apoVs inhibited osteogenesis of MSCs in vivo.

\section{MiR155 promoted adipogenesis of MSCs cultured with macrophage-derived apoVs.}

Several studies have shown the contents of EVs included mRNAs, miRNAs, ncRNAs, protein and lipids. MiRNAs account for about half of the total RNAs of EVs, and play a key role in the transfer of biomolecules to recipient cells and cell-to-cell communication(32-34). The content of apoVs was higher than that of exosomes, and the content of miRNA in apoptotic vesicles was much higher than that of exosomes (Fig.S3). The change of miR155 in apoVs was highly significant, indicating that miR155 was highly enriched in apoVs (Fig. 6A). Next, we transfected macrophages with inhibitor-negative control (inhiNC), inhibitor-miR155 (inhi-miR155), mimics-negative control (miR-NC) and mimics-miR155 (miR155), the expression levels of miR155 could be significantly decreased by (inhi-miR155) or increased by (miR155) in macrophages, and miR155 expression levels decreased or increased more significantly in corresponding apoVs: apoVs (inhi-NC), apoVs (inhi-miR155), apoVs (miR-NC) and apoVs (miR155), 
respectively (Fig. 6B). Subsequently, apoVs (inhi-NC), apoVs (inhi-miR155), apoVs (miR-NC) and apoVs (miR155) were added to MSCs for adipogenic induction. The results showed that the adipogenesis of MSCs was decreased in apoVs (inhi-miR155) group compared to the apoVs (inhi-NC) group. In addition, the adipogenesis ability of MSCs was improved in the apoVs (miR155) group compared to the apoVs (miR-NC) group (Fig. 6C, 6D). Collectively, these results showed that miR155 promoted adipogenic differentiation of MSCs cultured with macrophage-derived apoVs.

\section{MiR155 inhibited osteogenesis of MSCs cultured with macrophage-derived apoVs.}

In order to further clarify the role of miR155 in macrophage-derived apoVs in regulating the osteogenesis of MSCs, we cultured MSCs with apoVs (inhi-NC), apoVs (inhi-miR155), apoVs (miR-NC) and apoVs (miR155) in OM. The ALP staining and quantification showed that the osteogenesis of MSCs was upregulated in the apoVs (inhi-miR155) group compared with apoVs (inhi-NC) group, while the osteogenesis of MSCs was decreased in apoVs (miR155) group than the apoVs (miR-NC) group (Fig. 7A, 7B). ARS staining and quantification confirmed these results (Fig. 7C, 7D). Therefore, our results suggested that miR155 could regulate osteogenesis and adipogenesis of MSCs in the presence of macrophage-derived apoVs.

\section{MiR155 regulated adipogenic and osteogenic differentiation of MSCs cultured with macrophage-derived apoVs via the SMAD2 signaling pathway.}

SMAD2 was a target gene of miR155(35, 36), and enhancement of the SMAD2 signaling pathway increased osteogenic differentiation and inhibited adipogenic differentiation(37-39). We cultured MSCs with apoVs (inhi-NC), apoVs (inhi-miR155), apoVs (miR-NC) and apoVs (miR155) in AM and OM, then used western blot analysis to clarify the changes of SMAD2 pathway proteins (Fig. 8A, 8B). In the apoVs (inhi-miR155) group, the SMAD2 signaling pathway was up-regulated compared to the apoVs (inhi-NC) group. Moreover, the SMAD2 signaling pathway was down-regulated in the apoVs (miR155) group compared to the apoVs (miR-NC) group. Our data suggested that miR155 regulated adipogenic and osteogenic differentiation of MSCs cultured with macrophage-derived apoVs via the SMAD2 signaling pathway.

\section{Discussion}

Macrophages played important roles in stem cell survival and tissue repair(40). Different polarization states of macrophages have different effects on stem cell differentiation. In 2018, He et al. reported that conditioned medium of M1 macrophages could promote the adipogenesis of bone marrow mesenchymal stem cells (BMMSCs), whereas conditioned medium of M0 and M2 macrophages could promote the osteogenesis of BMMSCs(41). In 2020, Ma et al. showed that macrophages could inhibit the adipogenic differentiation of hASCs in vitro(42). Furthermore, the stem cell - macrophage interaction was associated with the development of several adipose related diseases(43-45). These studies suggested that macrophages could regulate the differentiation of MSCs via different molecular mechanisms. However, the effect of macrophage derived apoVs on the multidirectional differentiation of stem cells was unclear. 
In this study, we detected the role of macrophage-derived apoVs in the fate commitment of MSCs. We investigated that apoptotic macrophages could secrete a large number of apoVs which had a cup-shaped morphology and the diameter was under $1 \mu \mathrm{m}$. The content of apoptotic vesicles was higher than that of exosomes. ApoVs were ingested by MSCs and impacted multi-differentiation. In addition, we detected that macrophage-derived apoVs could promote the adipogenesis and inhibit osteogenesis of MSCs both in vitro and in vivo.

EVs secreted by cells contain a large number of miRNAs, which can be transported to recipient cells and change the expression of target genes(46-48). Although specific miRNAs have no clear physiological functions, miRNAs are involved in almost all biological processes. In 2017, Gu et al. reported that miR155 inhibited osteogenesis by targeting the positive regulation of osteogenesis factors(49). In 2019, Mao Z et al. have identified that miR155 was highly expressed in osteoporotic patients and inhibited osteoclast activation and bone resorption(50). Ying et al. found that miR155 was overexpressed in obese adipose tissue macrophage exosomes, and miR155-KO animals were insulin sensitive and glucose tolerant(51). SMAD2 was a predicted target gene of miR155(35), and the SMAD2 signaling pathway could regulate the osteogenesis and adipogenesis(52). We found that apoVs were enriched in miR155. ApoVs could be taken up by MSCs, and the contents of the apoVs could be transferred to stem cells. In addition, miR155 regulated adipogenic and osteogenic differentiation of MSCs cultured with macrophage-derived apoVs via the SMAD2 signaling pathway. Therefore, macrophage-derived apoVs could regulate the osteogenesis and adipogenesis of MSCs through miR155, suggesting a novel tissue engineering method based on multidirectional differentiation of stem cells.

Soft tissue defect or depression caused by trauma, infection, operation or congenital malformation is very common in clinic. Adipose tissue engineering can repair soft tissue defects, correct contour deformation, and provide solutions for tissue reconstruction after soft tissue trauma(53-57). Adipogenic differentiation of stem cells plays an important role in the construction of adipose tissue engineering and the treatment of soft tissue defects $(58,59)$. Our results showed that macrophage-derived apoVs could promote stem cell adipogenesis through miR155, which suggested that apoVs could be used as miRNA carriers to treat soft tissue defects. Thus, macrophage-derived apoVs could provide a new soft tissue method.

Overall, our study still has some limitations. Further analysis of binding of miR155 and SMAD2 by dual luciferase assay is needed. In future research, we plan to explore the influence of apoVs derived from M1 and M2 polarized macrophages on stem cell differentiation. Finally, further research is needed to enable clinical application of apoVs in tissue engineering.

\section{Conclusion}

Our study found that macrophage-derived apoVs could regulate osteogenesis and adipogenesis of MSCs through miR155. ApoVs could transport miRNAs and regulate stem cell multidirectional differentiation, 
which provides a novel method for tissue engineering based on multidirectional differentiation of stem cells.

\section{Abbreviations}

\begin{tabular}{|ll|}
\hline ALP & Alkaline phosphatase \\
AM & Adipogenic medium \\
\hline ApoVs & Apoptotic vesicles \\
\hline ARS & Alizarin Red S \\
\hline BGLAP & Bone gamma-carboxyglutamic acid-containing protein \\
\hline BMMSCs & Bone marrow mesenchymal stem cells \\
\hline C/EBPa & CCAAT/enhancer-binding protein a \\
\hline EVs & Extracellular vesicles \\
\hline FBS & Fetal bovine serum \\
\hline GAPDH & Glyceraldehyde3-phosphate dehydrogenase \\
\hline hASCs & Human adipose-derived stem cells \\
\hline H\&E & Hematoxylin and eosin \\
\hline inhibitor & Inhibitor-miR155 \\
\hline inhi-NC & Inhibitor - negative control \\
\hline mimics & Mimics-miR155 \\
\hline miR155 & MicroRNA155 \\
\hline miR-NC & Mimics-negative control \\
\hline MSCs & Mesenchymal stem cells \\
\hline NTA & Nanoparticle tracking analysis \\
\hline OM & Osteogenic medium \\
\hline PM & Proliferation medium \\
\hline PPARY & Peroxisome proliferator activated receptor Y \\
\hline RT-qPCR & Real-time quantitative polymerase chain reaction \\
\hline STS & Staurosporine \\
\hline TEM & Transmission electron microscopy \\
\hline
\end{tabular}




\section{Declarations}

- Acknowledgements

The authors are grateful to $\mathrm{Dr}$. Si Chen.

- Funding

This study was supported by grants from the Beijing Natural Science Foundation-Haidian Original Innovation Joint Fund (No. L182006 to YZ), Research Unit of Precision Pathologic Diagnosis in Tumors of the Oral and Maxillofacial Regions, Chinese Academy of Medical Sciences (No. $2019 \mathrm{RU} 034$ to YZ), the National Natural Science Foundation of China (No. 82170929 and 81970908 to YL)

- Availability of data and materials

The authors confirm that all data underlying the findings are fully available.

- Authors' contributions

$\mathrm{YZ}$ and $\mathrm{XZ}$ were responsible for the conception and design, collection and/or assembly of data, data analyses and interpretation, and manuscript writing. $\mathrm{RG}$ and $\mathrm{HL}$ were responsible for the collection and/or assembly of data and data analyses and interpretation in the animal experiments. KY, YS and XL were responsible for the collection and/or assembly of data and data analyses in the molecular biology experiments. $Y L$ and $Y Z$ was responsible for the conception and design, financial support, and manuscript writing. All authors read and approved the final manuscript.

- Ethics approval and consent to participate

This study was carried out in strict accordance with the recommendations of the Guide for the Care and Use of Laboratory Animals of the National Institutes of Health. The protocol was approved by the Institutional Animal Care and Use Committee of the Peking University Health Science Center (approval no. LA2021006). All surgeries were performed under anesthesia, and all efforts were made to minimize animal suffering.

- Consent for publication

Not applicable

- Competing interests

The authors declare that they have no competing interests.

\section{References}

1. Langer R, Vacanti J. Advances in tissue engineering. Journal of pediatric surgery. 2016;51(1):8-12. 
2. Marx V. Tissue engineering: Organs from the lab. Nature. 2015;522(7556):373-7.

3. Griffith LG, Naughton G. Tissue engineering-current challenges and expanding opportunities. Science. 2002;295(5557):1009-14.

4. Liu H, Cao T. Dental application potential of mesenchymal stromal cells and embryonic stem cells. Chin J Dent Res. 2010;13(2):95-103.

5. Gentile P, Sterodimas A, Calabrese C, Garcovich S. Systematic review: Advances of fat tissue engineering as bioactive scaffold, bioactive material, and source for adipose-derived mesenchymal stem cells in wound and scar treatment. Stem cell research \& therapy. 2021;12(1):318.

6. Fahimipour F, Dashtimoghadam E, Mahdi Hasani-Sadrabadi M, Vargas J, Vashaee D, Lobner DC, et al. Enhancing cell seeding and osteogenesis of MSCs on 3D printed scaffolds through injectable BMP2 immobilized ECM-Mimetic gel. Dental materials : official publication of the Academy of Dental Materials. 2019;35(7):990-1006.

7. Aravamudhan A, Ramos DM, Nip J, Subramanian A, James R, Harmon MD, et al. Osteoinductive small molecules: growth factor alternatives for bone tissue engineering. Curr Pharm Des. 2013;19(19):3420-8.

8. Atasoy-Zeybek A, Kose GT. Gene Therapy Strategies in Bone Tissue Engineering and Current Clinical Applications. Adv Exp Med Biol. 2018;1119:85-101.

9. Bianco P, Robey PG. Stem cells in tissue engineering. Nature. 2001;414(6859):118-21.

10. Howard D, Buttery LD, Shakesheff KM, Roberts SJ. Tissue engineering: strategies, stem cells and scaffolds. J Anat. 2008;213(1):66-72.

11. Zhu Y, Zhang P, Gu RL, Liu YS, Zhou YS. Origin and Clinical Applications of Neural Crest-Derived Dental Stem Cells. Chin J Dent Res. 2018;21(2):89-100.

12. Liu G, Chen X. Isolating and Characterizing Adipose-Derived Stem Cells. Methods Mol Biol. 2018;1842:193-201.

13. Zhu Y, Zhang X, Gu R, Liu X, Wang S, Xia D, et al. LAMA2 regulates the fate commitment of mesenchymal stem cells via hedgehog signaling. Stem cell research \& therapy. 2020;11(1):135.

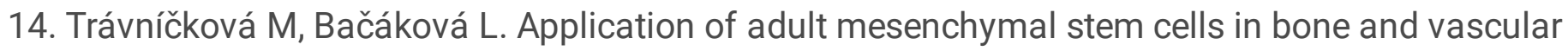
tissue engineering. Physiol Res. 2018;67(6):831-50.

15. Ye X, Zhang P, Xue S, Xu Y, Tan J, Liu G. Adipose-derived stem cells alleviate osteoporosis by enhancing osteogenesis and inhibiting adipogenesis in a rabbit model. Cytotherapy. 2014;16(12):1643-55.

16. Koch TG, Berg LC, Betts DH. Current and future regenerative medicine - principles, concepts, and therapeutic use of stem cell therapy and tissue engineering in equine medicine. Can Vet $\mathrm{J}$. 2009;50(2):155-65.

17. Miao X, Leng X, Zhang Q. The Current State of Nanoparticle-Induced Macrophage Polarization and Reprogramming Research. Int J Mol Sci. 2017;18(2). 
18. Li X, He XT, Kong DQ, Xu XY, Wu RX, Sun LJ, et al. M2 Macrophages Enhance the Cementoblastic Differentiation of Periodontal Ligament Stem Cells via the Akt and JNK Pathways. Stem cells (Dayton, Ohio). 2019;37(12):1567-80.

19. Vogel DY, Heijnen PD, Breur M, de Vries HE, Tool AT, Amor S, et al. Macrophages migrate in an activation-dependent manner to chemokines involved in neuroinflammation. Journal of neuroinflammation. 2014;11:23.

20. Li Q, Zheng M, Liu Y, Sun W, Shi J, Ni J, et al. A pathogenetic role for M1 macrophages in peritoneal dialysis-associated fibrosis. Molecular immunology. 2018;94:131-9.

21. Kang M, Huang CC, Lu Y, Shirazi S, Gajendrareddy P, Ravindran S, et al. Bone regeneration is mediated by macrophage extracellular vesicles. Bone. 2020;141:115627.

22. Xiong Y, Chen L, Yan C, Zhou W, Yu T, Sun Y, et al. M2 Macrophagy-derived exosomal miRNA-5106 induces bone mesenchymal stem cells towards osteoblastic fate by targeting salt-inducible kinase 2 and 3. Journal of nanobiotechnology. 2020;18(1):66.

23. Xia Y, He XT, Xu XY, Tian BM, An Y, Chen FM. Exosomes derived from M0, M1 and M2 macrophages exert distinct influences on the proliferation and differentiation of mesenchymal stem cells. PeerJ. 2020;8:e8970.

24. Cheng X, Ferrell JE, Jr. Apoptosis propagates through the cytoplasm as trigger waves. Science. 2018;361(6402):607-12.

25. Radi E, Formichi P, Battisti C, Federico A. Apoptosis and oxidative stress in neurodegenerative diseases. Journal of Alzheimer's disease : JAD. 2014;42 Suppl 3:S125-52.

26. Guo Y, Tan J, Miao Y, Sun Z, Zhang Q. Effects of Microvesicles on Cell Apoptosis under Hypoxia. Oxidative medicine and cellular longevity. 2019;2019:5972152.

27. Caruso S, Poon IKH. Apoptotic Cell-Derived Extracellular Vesicles: More Than Just Debris. Frontiers in immunology. 2018;9:1486.

28. Zheng C, Sui B, Zhang X, Hu J, Chen J, Liu J, et al. Apoptotic vesicles restore liver macrophage homeostasis to counteract type 2 diabetes. Journal of extracellular vesicles. 2021;10(7):e12109.

29. Liu D, Kou X, Chen C, Liu S, Liu Y, Yu W, et al. Circulating apoptotic bodies maintain mesenchymal stem cell homeostasis and ameliorate osteopenia via transferring multiple cellular factors. Cell research. 2018;28(9):918-33.

30. Liu H, Liu S, Qiu X, Yang X, Bao L, Pu F, et al. Donor MSCs release apoptotic bodies to improve myocardial infarction via autophagy regulation in recipient cells. Autophagy. 2020;16(12):2140-55.

31. Li W, Liu Y, Zhang P, Tang Y, Zhou M, Jiang W, et al. Tissue-Engineered Bone Immobilized with Human Adipose Stem Cells-Derived Exosomes Promotes Bone Regeneration. ACS applied materials \& interfaces. 2018;10(6):5240-54.

32. Abels ER, Breakefield XO. Introduction to Extracellular Vesicles: Biogenesis, RNA Cargo Selection, Content, Release, and Uptake. Cellular and molecular neurobiology. 2016;36(3):301-12. 
33. Elsharkasy OM, Nordin JZ, Hagey DW, de Jong OG, Schiffelers RM, Andaloussi SE, et al. Extracellular vesicles as drug delivery systems: Why and how? Adv Drug Deliv Rev. 2020;159:332-43.

34. Yu X, Odenthal M, Fries JW. Exosomes as miRNA Carriers: Formation-Function-Future. Int J Mol Sci. 2016;17(12).

35. Louafi F, Martinez-Nunez RT, Sanchez-Elsner T. MicroRNA-155 targets SMAD2 and modulates the response of macrophages to transforming growth factor-\{beta\}. J Biol Chem. 2010;285(53):4132836.

36. Li CL, Nie H, Wang M, Su LP, Li JF, Yu YY, et al. microRNA-155 is downregulated in gastric cancer cells and involved in cell metastasis. Oncology reports. 2012;27(6):1960-6.

37. Wu D, Yin L, Sun D, Wang F, Wu Q, Xu Q, et al. Long noncoding RNA TUG1 promotes osteogenic differentiation of human periodontal ligament stem cell through sponging microRNA-222-3p to negatively regulate Smad2/7. Arch Oral Biol. 2020;117:104814.

38. Zheng W, Chen Q, Zhang Y, Xia R, Gu X, Hao Y, et al. BMP9 promotes osteogenic differentiation of SMSCs by activating the JNK/Smad2/3 signaling pathway. Journal of cellular biochemistry. 2020;121(4):2851-63.

39. Li H, Fan J, Fan L, Li T, Yang Y, Xu H, et al. MiRNA-10b Reciprocally Stimulates Osteogenesis and Inhibits Adipogenesis Partly through the TGF-B/SMAD2 Signaling Pathway. Aging and disease. 2018;9(6):1058-73.

40. Cai J, Feng J, Liu K, Zhou S, Lu F. Early Macrophage Infiltration Improves Fat Graft Survival by Inducing Angiogenesis and Hematopoietic Stem Cell Recruitment. Plastic and reconstructive surgery. 2018;141(2):376-86.

41. He XT, Li X, Yin Y, Wu RX, Xu XY, Chen FM. The effects of conditioned media generated by polarized macrophages on the cellular behaviours of bone marrow mesenchymal stem cells. Journal of cellular and molecular medicine. 2018;22(2):1302-15.

42. Ma H, Li YN, Song L, Liu R, Li X, Shang Q, et al. Macrophages inhibit adipogenic differentiation of adipose tissue derived mesenchymal stem/stromal cells by producing pro-inflammatory cytokines. Cell \& bioscience. 2020;10:88.

43. Rosen ED, Spiegelman BM. What we talk about when we talk about fat. Cell. 2014;156(1-2):20-44.

44. Zheng C, Yang Q, Cao J, Xie N, Liu K, Shou P, et al. Local proliferation initiates macrophage accumulation in adipose tissue during obesity. Cell death \& disease. 2016;7(3):e2167.

45. Zheng C, Yang Q, Xu C, Shou P, Cao J, Jiang M, et al. CD11b regulates obesity-induced insulin resistance via limiting alternative activation and proliferation of adipose tissue macrophages. Proc Natl Acad Sci U S A. 2015;112(52):E7239-48.

46. Dhondt B, Rousseau Q, De Wever O, Hendrix A. Function of extracellular vesicle-associated miRNAs in metastasis. Cell and tissue research. 2016;365(3):621-41.

47. Yoshiko Y, Minamizaki T. Emerging roles of microRNAs as extracellular vesicle cargo secreted from osteoblasts. Journal of oral biosciences. 2020;62(3):228-34. 
48. Li X, Ballantyne LL, Yu Y, Funk CD. Perivascular adipose tissue-derived extracellular vesicle miR-221$3 p$ mediates vascular remodeling. FASEB journal : official publication of the Federation of American Societies for Experimental Biology. 2019;33(11):12704-22.

49. Gu Y, Ma L, Song L, Li X, Chen D, Bai X. miR-155 Inhibits Mouse Osteoblast Differentiation by Suppressing SMAD5 Expression. Biomed Res Int. 2017;2017:1893520.

50. Mao Z, Zhu Y, Hao W, Chu C, Su H. MicroRNA-155 inhibition up-regulates LEPR to inhibit osteoclast activation and bone resorption via activation of AMPK in alendronate-treated osteoporotic mice. IUBMB life. 2019;71(12):1916-28.

51. Ying W, Riopel M, Bandyopadhyay G, Dong Y, Birmingham A, Seo JB, et al. Adipose Tissue Macrophage-Derived Exosomal miRNAs Can Modulate In Vivo and In Vitro Insulin Sensitivity. Cell. 2017;171(2):372-84.e12.

52. Gao L, Li SL, Li YK. Liraglutide Promotes the Osteogenic Differentiation in MC3T3-E1 Cells via Regulating the Expression of Smad2/3 Through PI3K/Akt and Wnt/ß-Catenin Pathways. DNA and cell biology. 2018;37(12):1031-43.

53. Suzuki K, Akita S, Yoshimoto H, Ohtsuru A, Hirano A, Yamashita S. Biological Features Implies Potential Use of Autologous Adipose-Derived Stem/Progenitor Cells in Wound Repair and Regenerations for the Patients with Lipodystrophy. Int J Mol Sci. 2019;20(21).

54. Xia Z, Guo X, Yu N, Zeng A, Si L, Long F, et al. The Application of Decellularized Adipose Tissue Promotes Wound Healing. Tissue Eng Regen Med. 2020;17(6):863-74.

55. Turner AE, Yu C, Bianco J, Watkins JF, Flynn LE. The performance of decellularized adipose tissue microcarriers as an inductive substrate for human adipose-derived stem cells. Biomaterials. 2012;33(18):4490-9.

56. Flynn L, Prestwich GD, Semple JL, Woodhouse KA. Adipose tissue engineering with naturally derived scaffolds and adipose-derived stem cells. Biomaterials. 2007;28(26):3834-42.

57. Xia P, Zhang K, Gong Y, Li G, Yan S, Yin J. Injectable Stem Cell Laden Open Porous Microgels That Favor Adipogenesis: In Vitro and in Vivo Evaluation. ACS applied materials \& interfaces. 2017;9(40):34751-61.

58. Karagoz H, Zor F, Goktas E, Gorantla VS. Adipogenesis for soft tissue reconstruction. Current Opinion in Organ Transplantation. 2019;24(5).

59. Blum C, Schlegelmilch K, Schilling T, Shridhar A, Rudert M, Jakob F, et al. Extracellular MatrixModified Fiber Scaffolds as a Proadipogenic Mesenchymal Stromal Cell Delivery Platform. ACS Biomaterials Science \& Engineering. 2019;5(12):6655-66.

\section{Tables}

Table 1 List of primers used in this study. 


\begin{tabular}{|lll|}
\hline Gene & Forward primer $\left(5^{\prime}\right.$ - $\left.^{\prime}\right)$ & Reverse primer $\left(5^{\prime}\right.$ - $\left.^{\prime}\right)$ \\
\hline ALP & GACCTCCTCGGAAGACACTC & TGAAGGGCTTCTTGTCTGTG \\
\hline BGLAP & AGCAAAGGTGCAGCCTTTGT & GCGCCTGGGTCTCTTCACT \\
\hline PPARY & CCGCCTCAGTGATTTAGGGC & GGGTCTGTAATCTGACTCTGTCC \\
\hline C/EBPa & GAGGAGCCTAAGGTAAGGAG & GTCATTTCGTTAAAGGCTGA \\
\hline GAPDH & GAAGGTGAAGGTCGGAGTC & GAAGATGGTGATGGGATTTC \\
\hline mmu-miR155 & GCTTCGGTTAATGCTAATCGTG & CAGAGCAGGGTCCGAGGTA \\
\hline mmu-U6 & CGCTTCGGCAGCACATATAC & TTCACGAATTTGCGTGTCATC \\
\hline
\end{tabular}

Table 2 List of miRNAs used in this study.

\begin{tabular}{|lll|}
\hline & Sense $\left(5^{\prime}-3^{\prime}\right)$ & Antisense $\left(5^{\prime}-3^{\prime}\right)$ \\
\hline mmu-miR155 & UUAAUGCUAAUUGUGAUAGGGGU & CCCUAUCACAAUUAGCAUUAAUU \\
\hline mmu-mimics-NC & UUGUACUACACAAAAGUACUG & GUACUUUUGUGUAGUACAAUU \\
\hline mmu-inhibitor-miR155 & ACCCCUAUCACAAUUAGCAUUAA & \\
\hline mmu-inhibitor-NC & CAGUACUUUUGUGUAGUACAA & \\
\hline
\end{tabular}

\section{Figures}


A

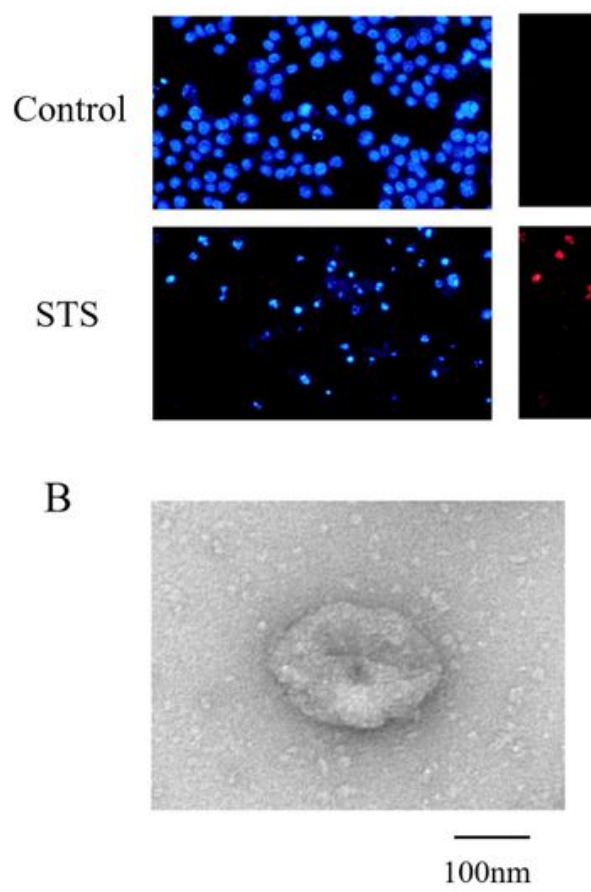

D
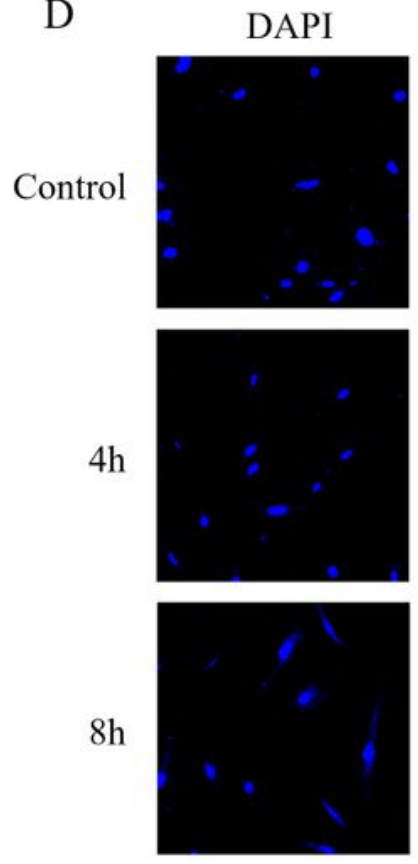

TUNEL
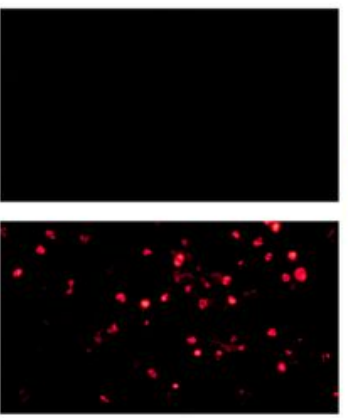

C

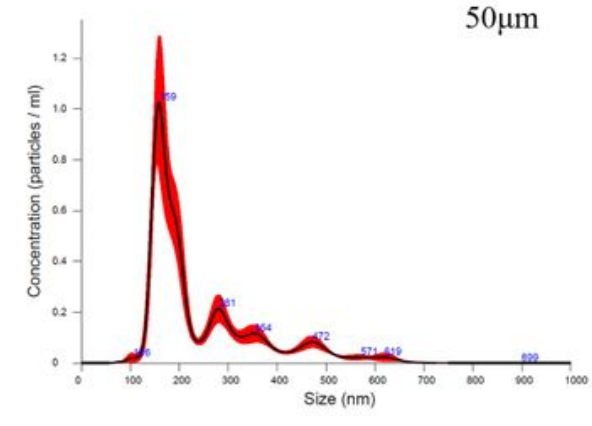

Phalloidin
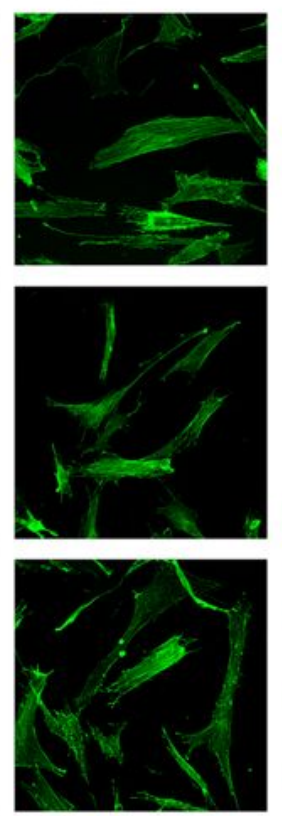

Merged
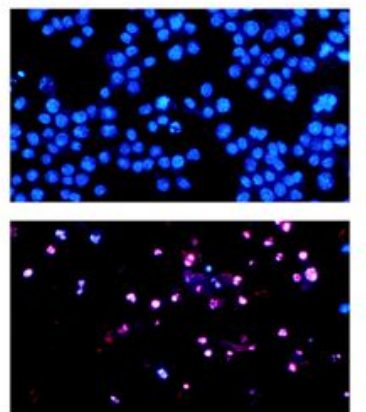

$\overline{50 \mu \mathrm{m}}$

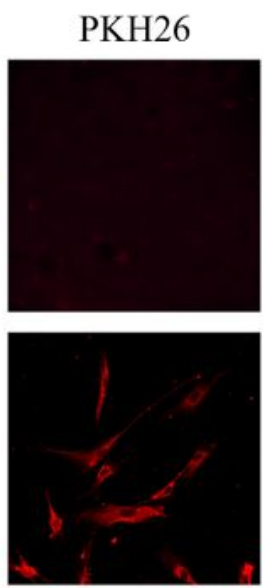

Merged
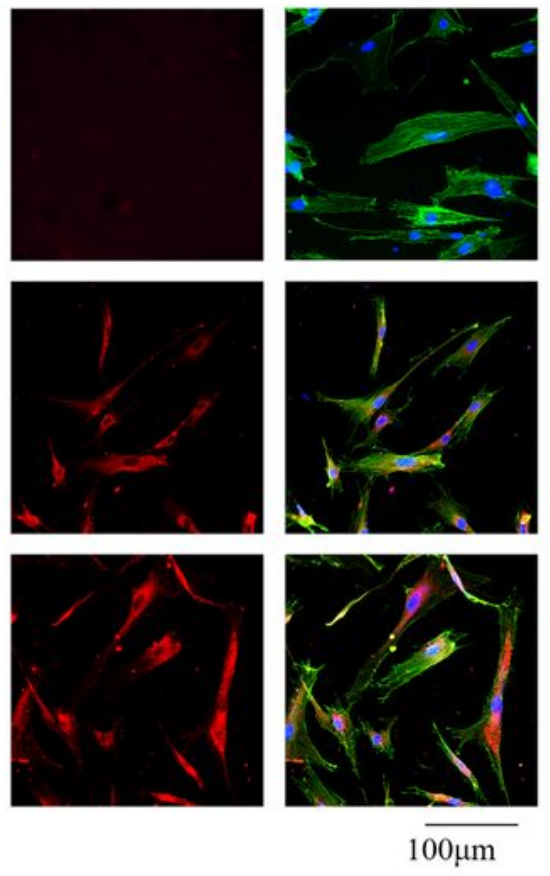

\section{Figure 1}

Characterization of macrophage-derived apoVs and apoVs uptake by MSCs. A. TUNEL staining results in different groups. TUNEL positive stained cells was red. B. Morphology of apoVs observed by transmission electron microscopy. C. Particle size distribution of apoVs measured by Nano Sight analysis: the mean size \pm SD of exosomes was $240.6 \pm 115 \mathrm{~nm}$. D. MSCs were incubated with PKH-26- 
labeled apoVs (red) for $4 \mathrm{~h}$ and $8 \mathrm{~h}$, respectively. The nuclei of MSCs were stained with DAPI (blue). The Factin of MSCs was stained with phalloidin (green).

A

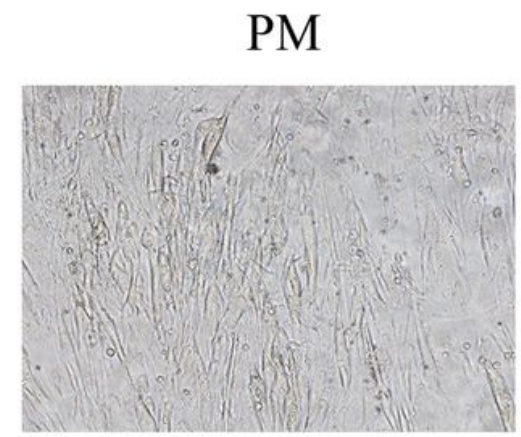

$\mathrm{B}$

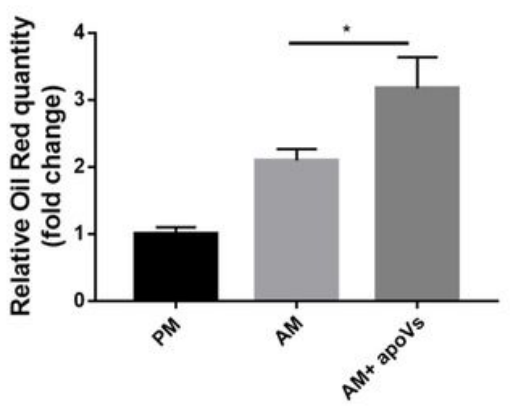

$\mathrm{D}$

\section{PPAR $\gamma$}

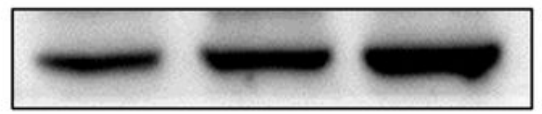

GAPDH

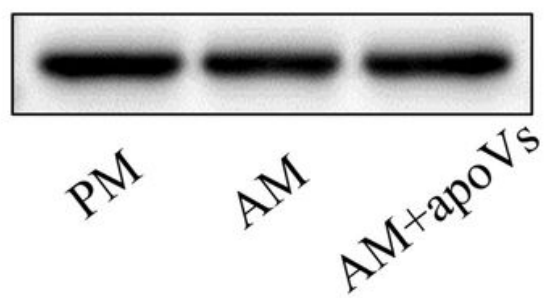

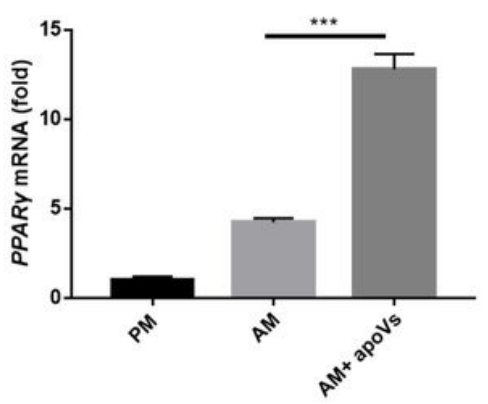

$\mathrm{AM}$

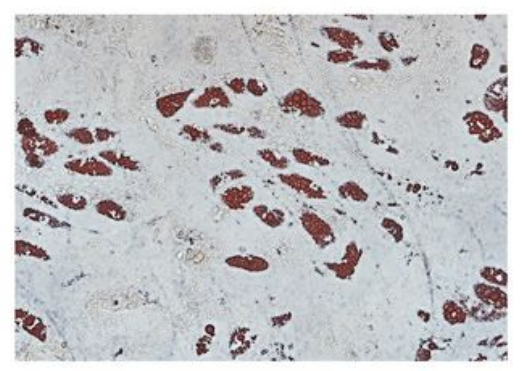

$\mathrm{C}$

$\mathrm{E}$

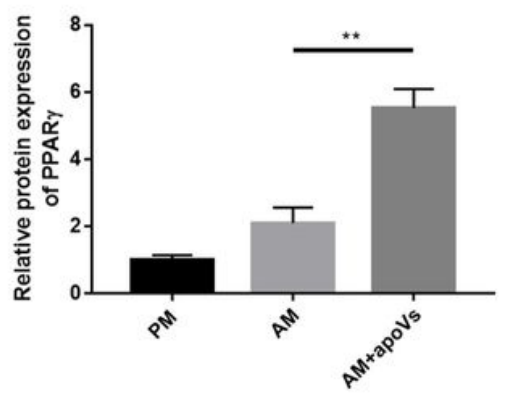

Figure 2

Macrophage-derived apoVs promoted adipogenesis of MSCs in vitro. A. Macrophage-derived apoVs promoted adipogenesis as indicated by oil red $\mathrm{O}$ staining. $\mathrm{B}$. Oil red $\mathrm{O}$ quantitative analysis showed that apoVs promoted adipogenesis. C. Macrophage-derived apoVs enhanced the mRNA expression of PPARY and C/EBPa detected by RT-qPCR. D. Western blot showed that the protein expression of PPARY was upregulated in the group treated with apoVs compared with the group without apoVs. E. Western blot quantification showed the same result as Figure 2D. ${ }^{*} P<0.05,{ }^{\star} * P<0.01, * \star \star P<0.001$. 
A

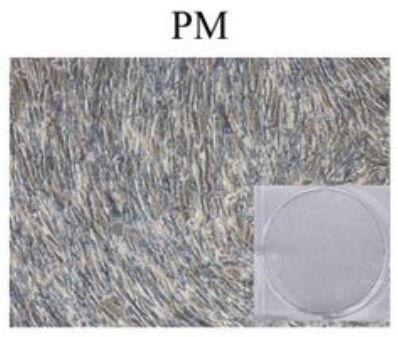

B

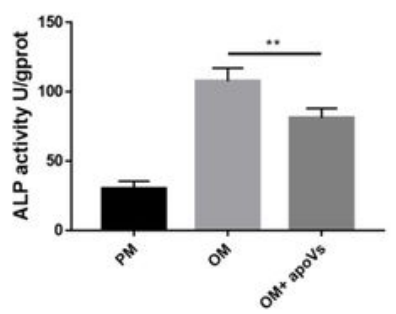

D

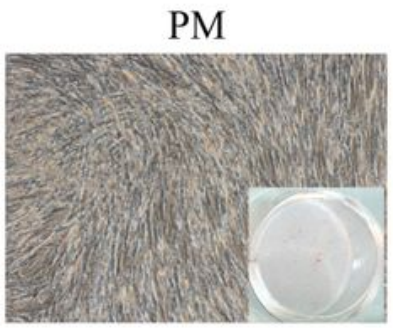

E

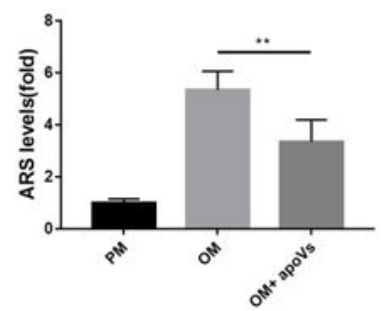

G

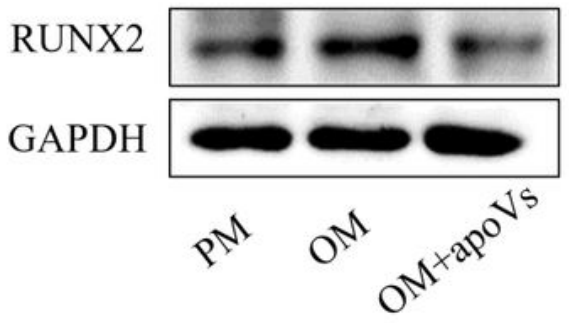

OM

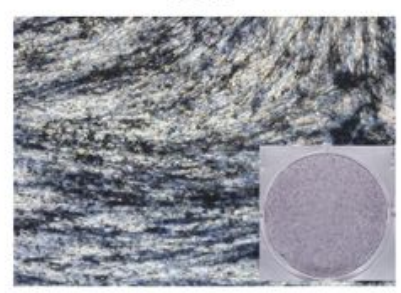

C

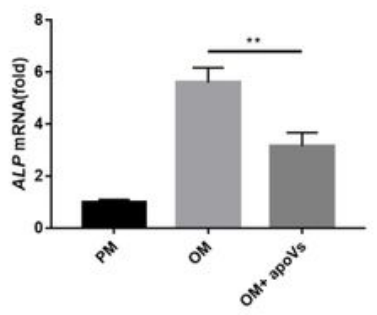

OM

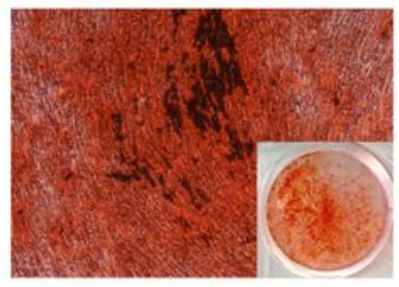

F

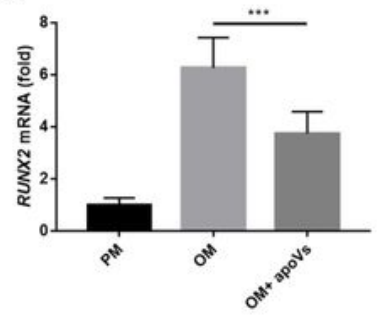

H
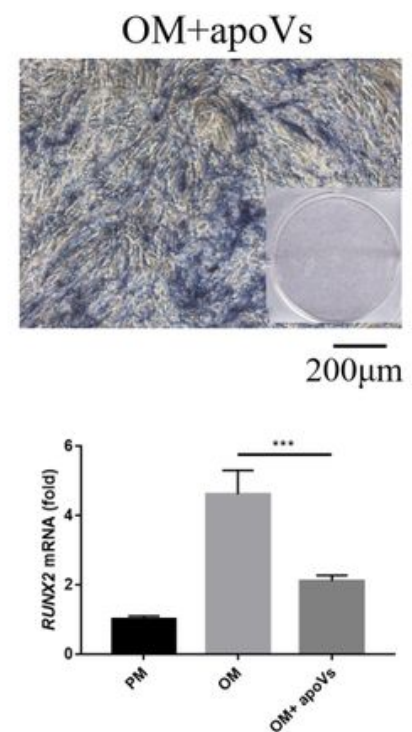

$\mathrm{OM}+\mathrm{apoVs}$

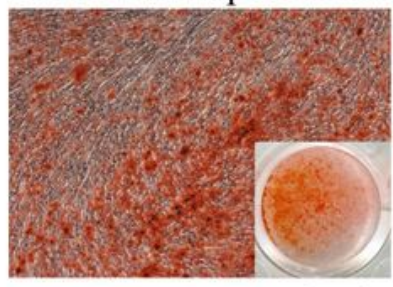

$200 \mu \mathrm{m}$
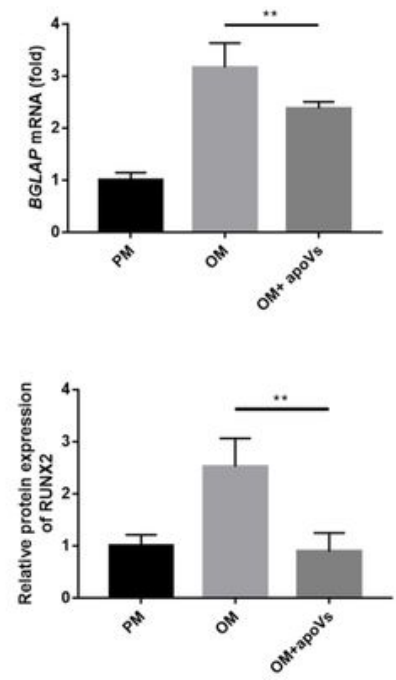

\section{Figure 3}

Macrophage-derived apoVs inhibited osteogenesis of MSCs in vitro. A. ApoVs inhibited osteogenic differentiation as indicated by ALP staining. B. ApoVs inhibited osteogenic differentiation as indicated by ALP quantification. C. ApoVs inhibited the mRNA expression of ALP and RUNX2 detected by RT-qPCR. D. ApoVs inhibited osteogenesis as indicated by ARS staining. E. ApoVs inhibited osteogenesis as indicated by ARS quantification. F. ApoVs reduced the mRNA expression of RUNX2 and BGLAP detected by RT- 
qPCR. G. Western blot showed that the protein expression of RUNX2 was down-regulated in the group treated with apoVs compared with the group without apoVs. H. Western blot quantification showed the same result as Figure 3G. ${ }^{\star *} \mathrm{P}<0.01, * \star * \mathrm{P}<0.001$.

A
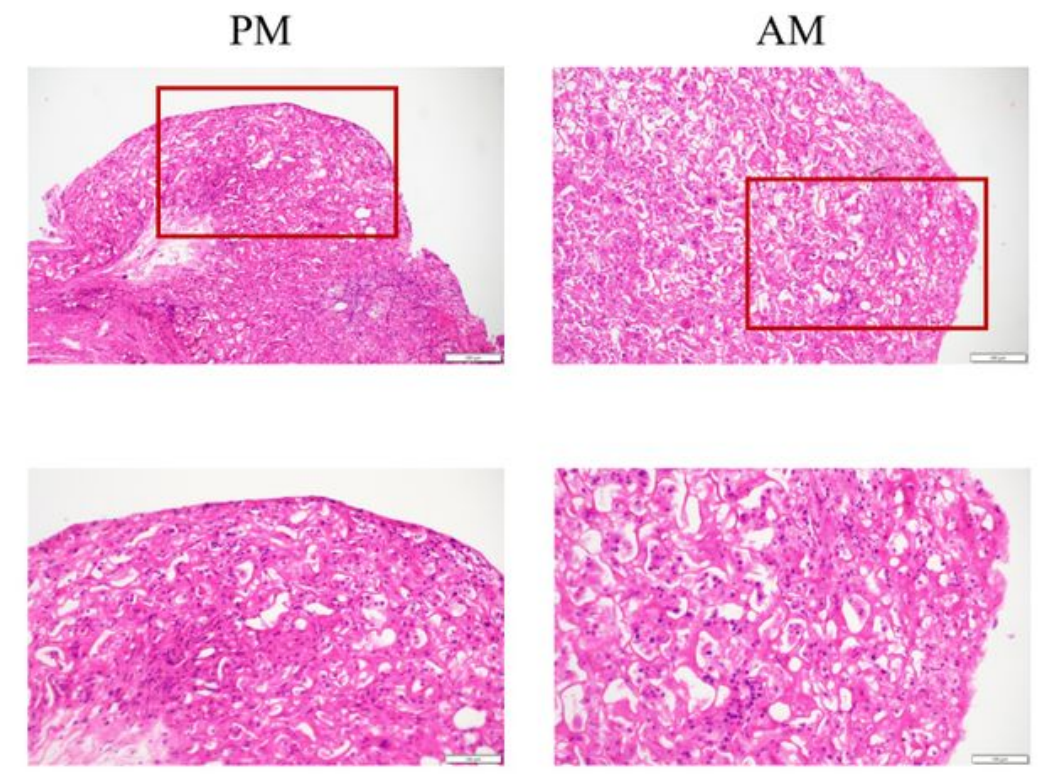

AM+apoVs

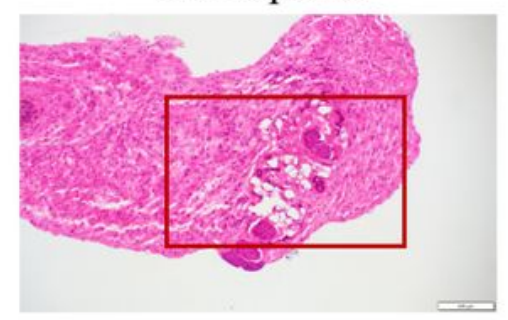

$20 \overline{\mu m}$

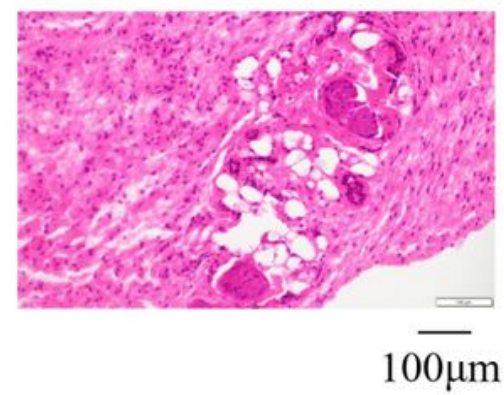

$\mathrm{AM}$

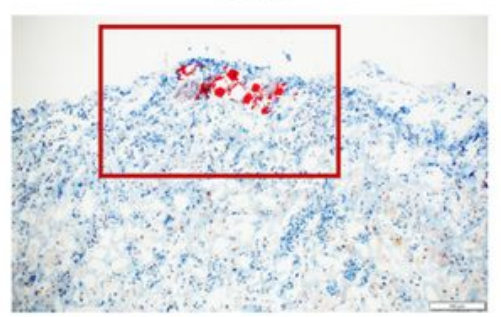

$\mathrm{AM}+\mathrm{apoVs}$
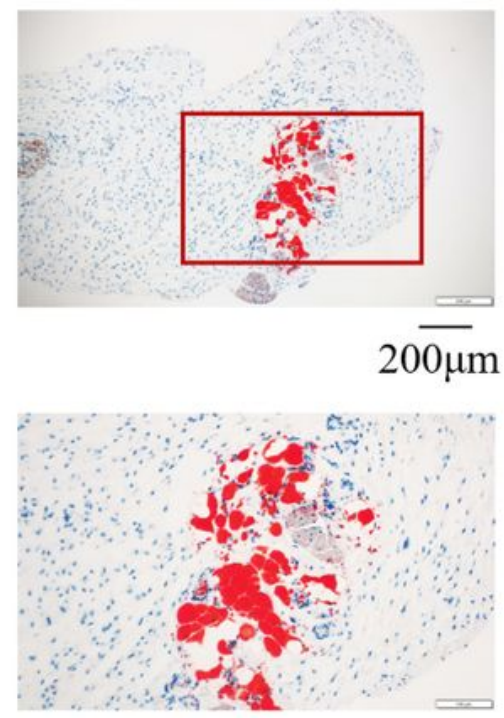

$100 \mu \mathrm{m}$

\section{Figure 4}

Macrophage-derived apoVs promoted adipogenesis of MSCs in vivo. A. H\&E staining of the PM, AM, and $\mathrm{AM}+$ apoVs groups of MSCs. B. Oil red O staining of the PM, AM, and AM + apoVs groups of MSCs. The 
red rectangles indicated the corresponding magnified areas.

A
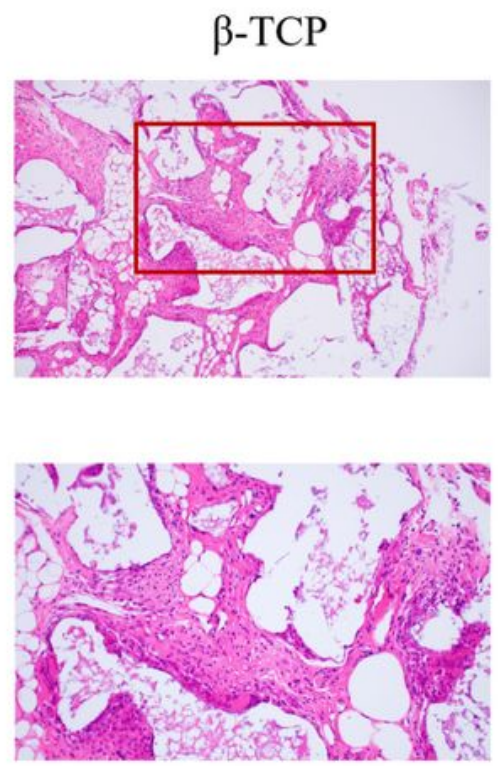

B
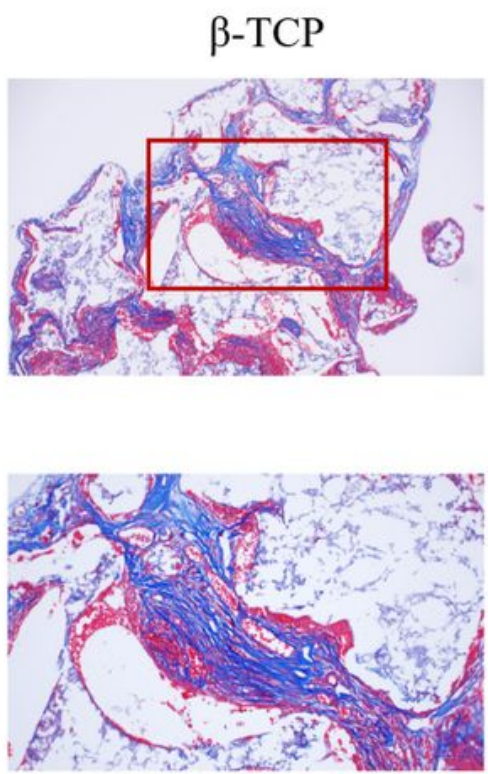

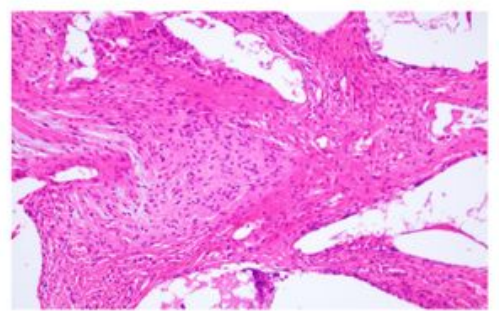

PM
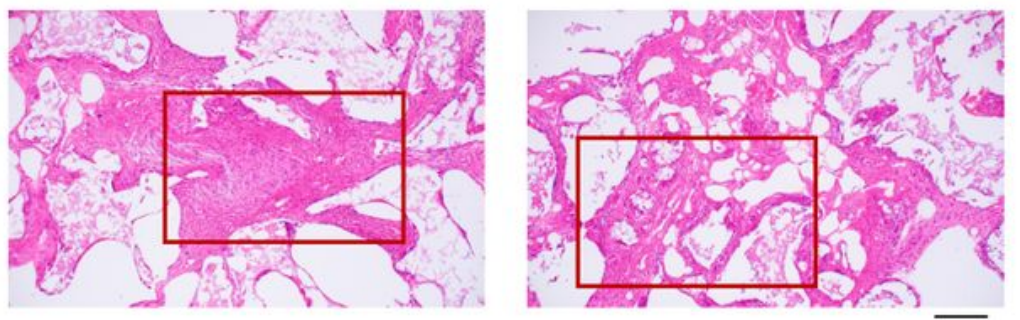

$200 \mu \mathrm{m}$

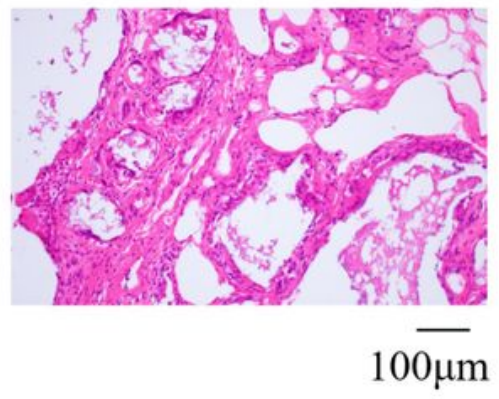

PM
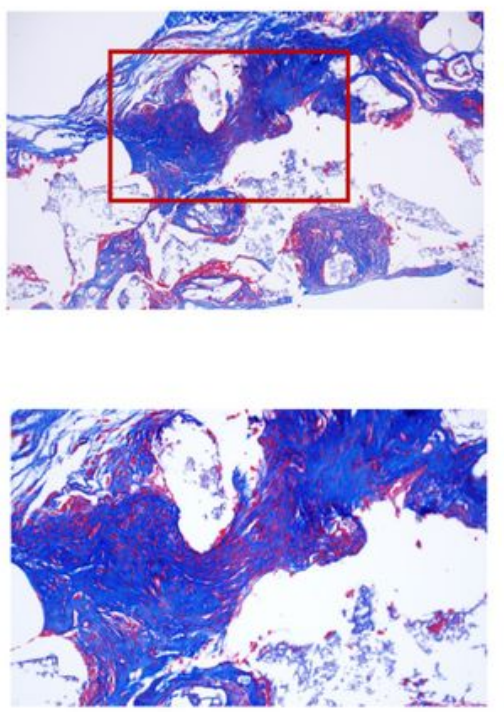

PM+apoVs
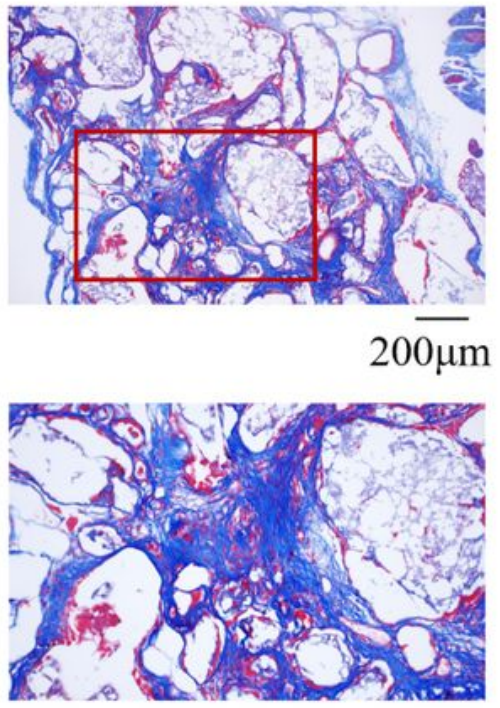

$100 \mu \mathrm{m}$

\section{Figure 5}

Macrophage-derived apoVs inhibited osteogenesis of MSCs in vivo. A. H\&E staining of the $\beta$-TCP, PM and $P M+$ apoVs groups. B. Masson staining of the $\beta-T C P, P M$ and PM + apoVs groups. The red rectangles indicated the corresponding magnified areas. 

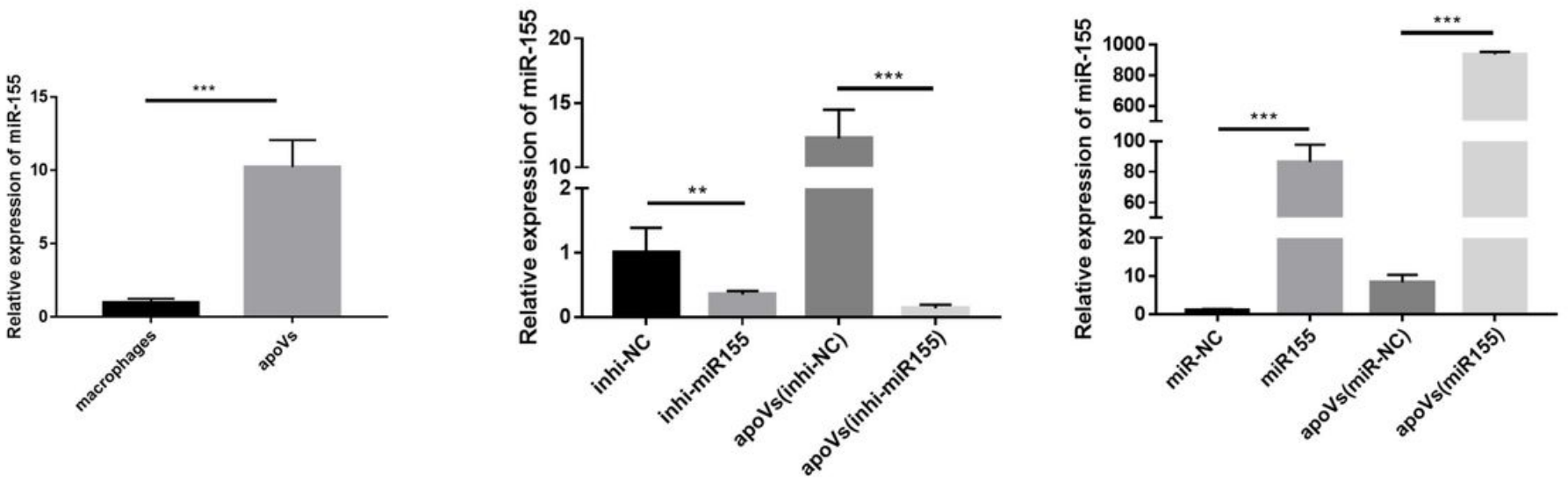

C

PM

AM

$\mathrm{AM}+$

$\mathrm{AM}+$

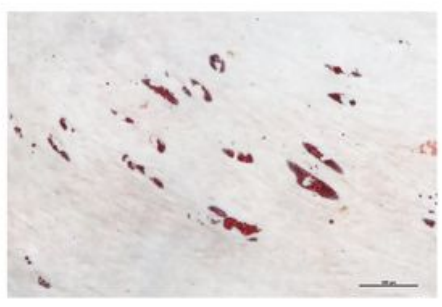

apoVs (inhibi-NC)

apoVs (inhi-miR155)
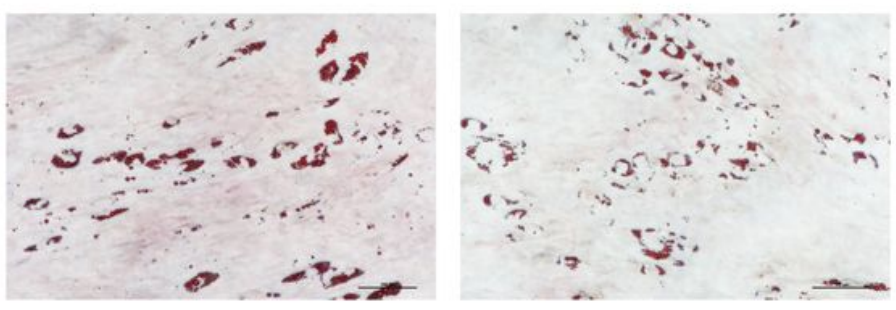

AM+

AM+

D apoVs (miR-NC)

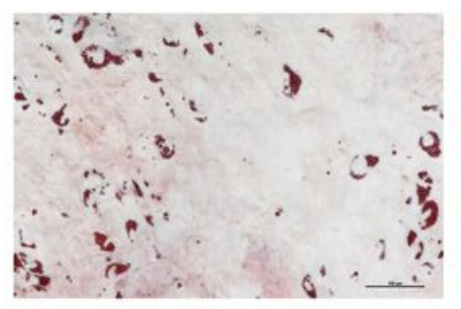
apoVs (miR155)
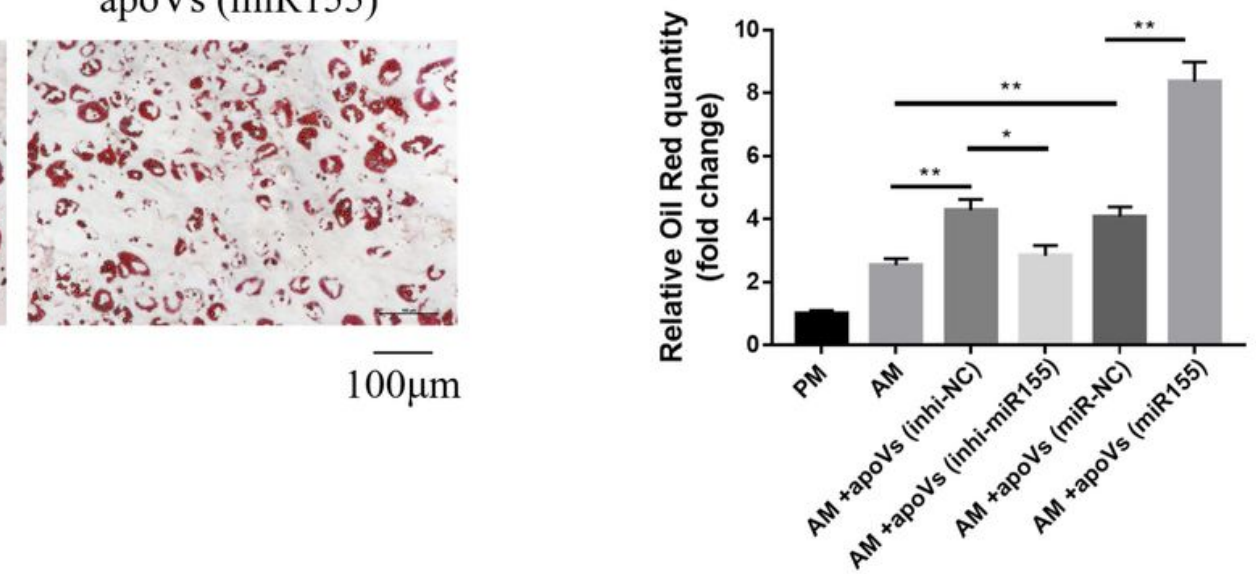

Figure 6

MiR155 promoted adipogenesis of MSCs cultured with macrophage-derived apoVs. A. Expression levels of miR155 in macrophages and apoVs indicated that miR155 were highly enriched in apoVs. B.

Expression levels of miR155 in macrophages transfected with inhi-NC, inhi-miR155, miR-NC and miR155 and corresponding apoVs. C. MSCs cultured in PM or AM treated with or without apoVs stained for oil red O. D. Quantification of oil red $0 .{ }^{*} P<0.05, * \star P<0.01, * \star * P<0.001$. 
A

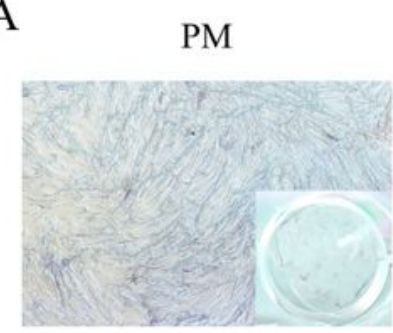

$\mathrm{OM}+$
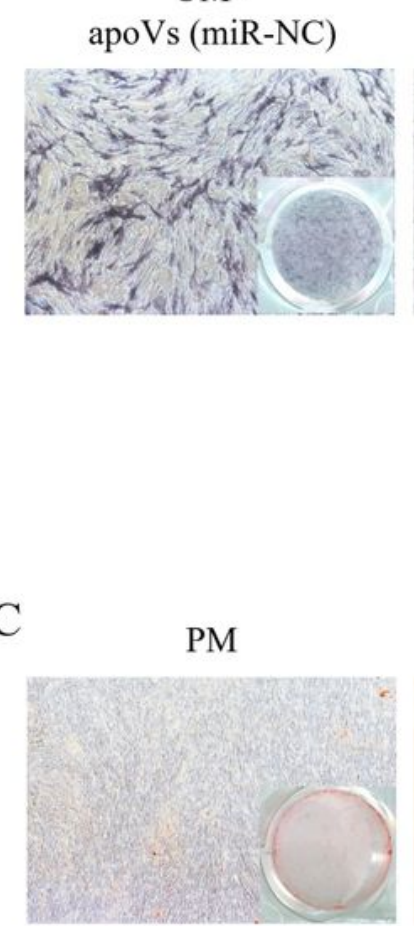

$\mathrm{OM}+$ apoVs (miR-NC)

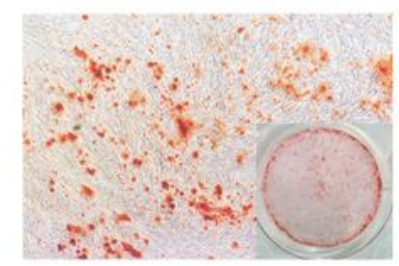

OM

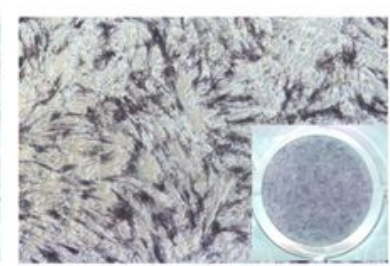

$\mathrm{OM}+$ apoVs (miR155)

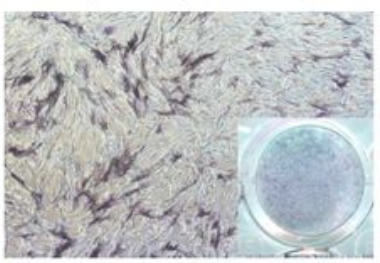

$20 \overline{\mu \mathrm{m}}$
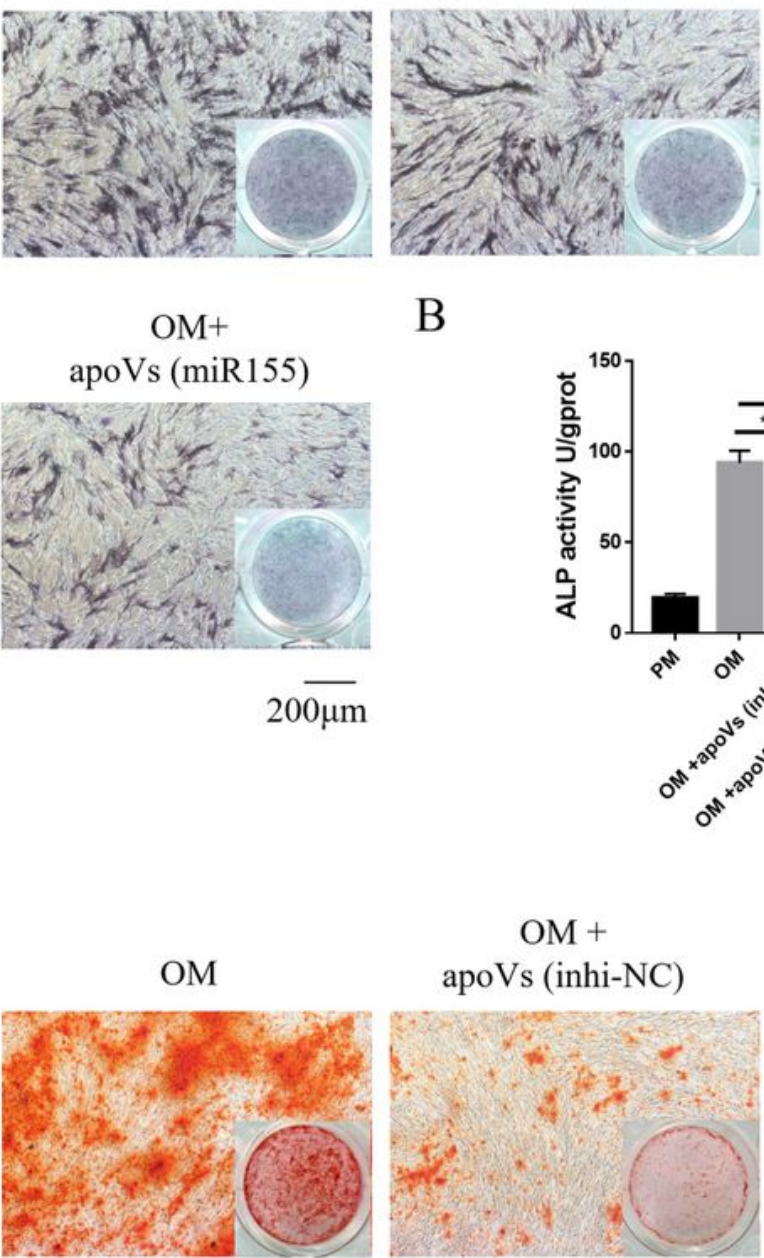

$\mathrm{OM}+$ apoVs (miR155)

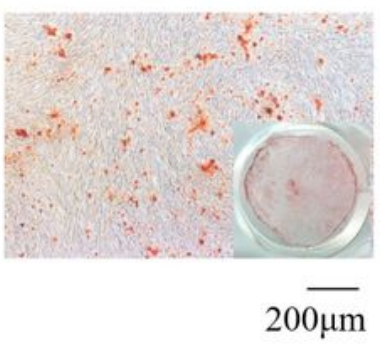

$\mathrm{OM}+$ apoVs (inhi-NC)

$\mathrm{B}$

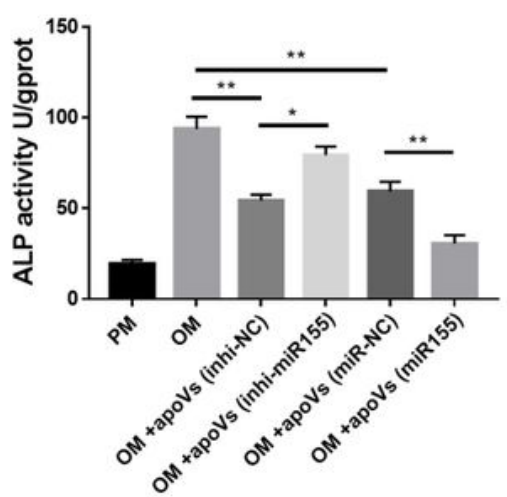

$\mathrm{OM}+$ apoVs (inhi-NC)

$\mathrm{OM}+$ apoVs (inhi-miR155)
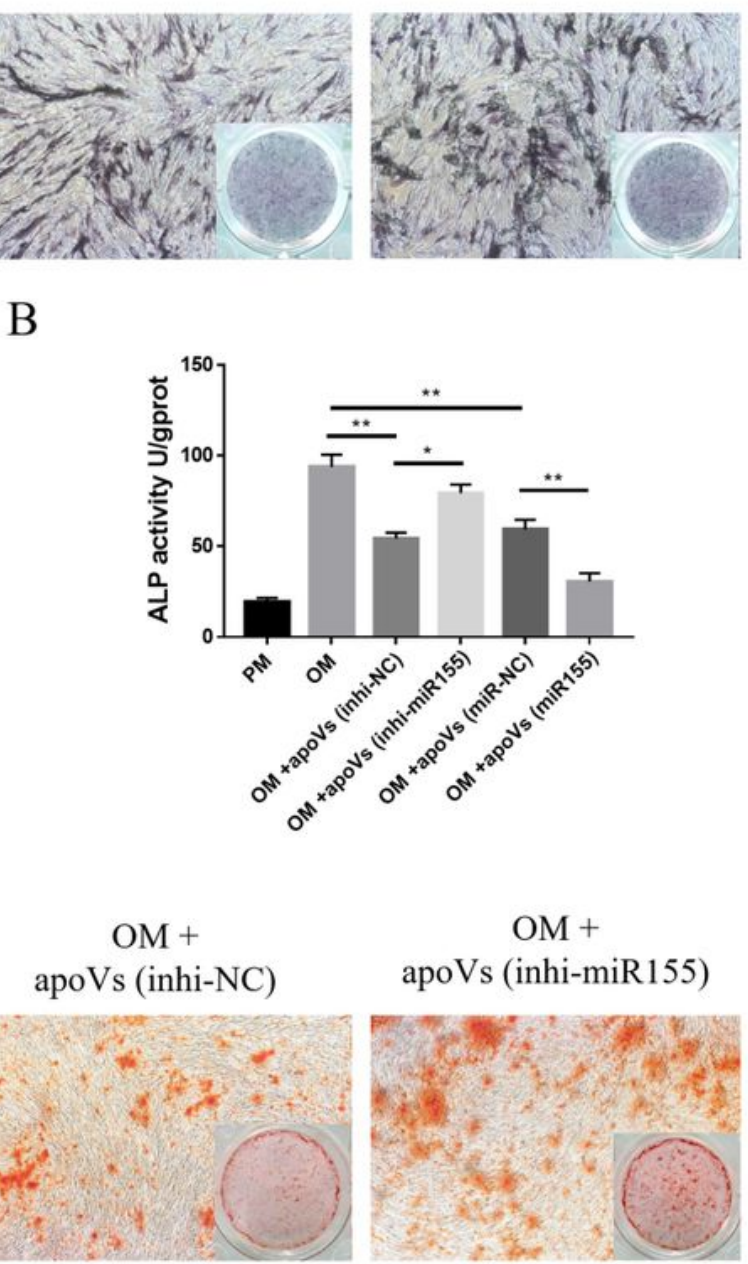
apoVs (inhi-miR155)

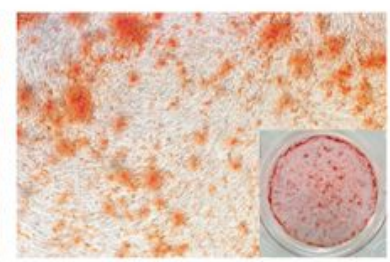

$\mathrm{D}$

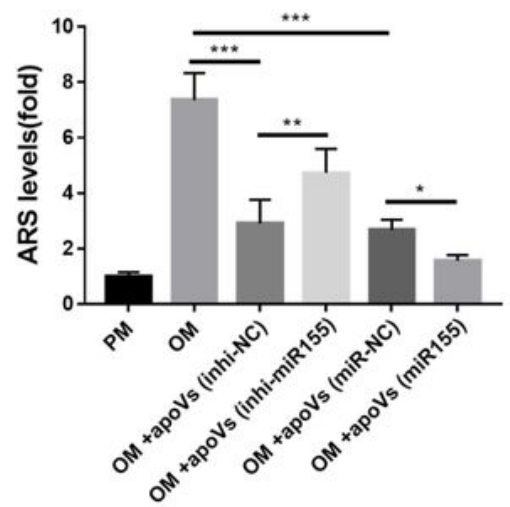

\section{Figure 7}

MiR155 inhibited osteogenesis of MSCs cultured with macrophage-derived apoVs. A. MSCs cultured in PM or OM treated with or without apoVs stained for ALP. B. Quantification of ALP. C. MSCs cultured in PM or OM treated with or without apoVs stained for ARS. D. Quantification of ARS. ${ }^{*} P<0.05,{ }^{\star *} P<0.01, \star \star * P$ $<0.001$. 
A

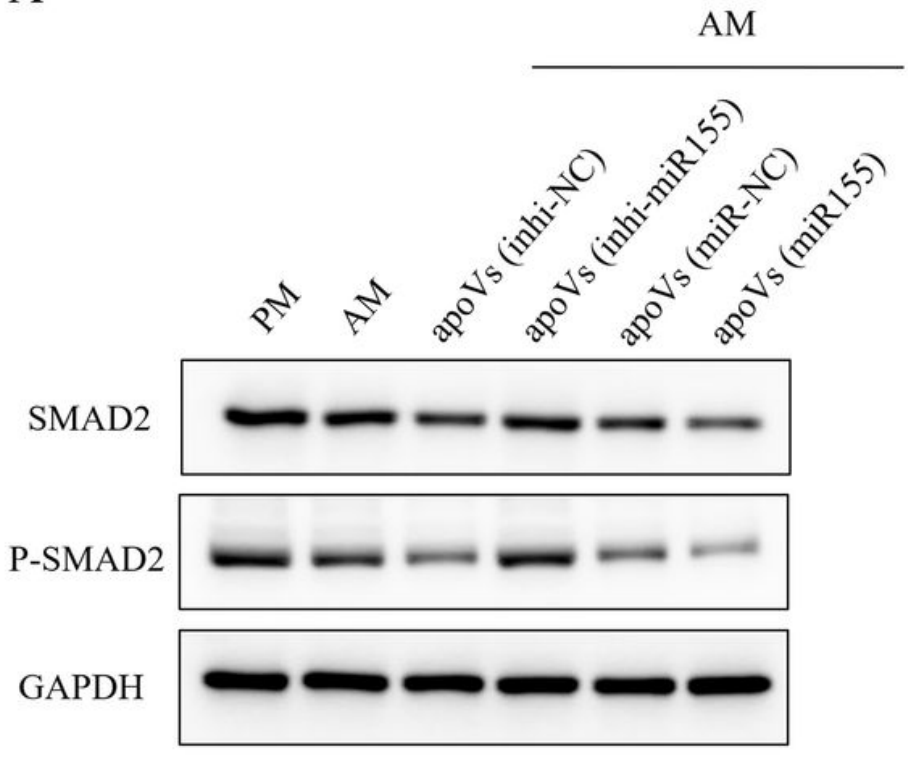

$\mathrm{B}$

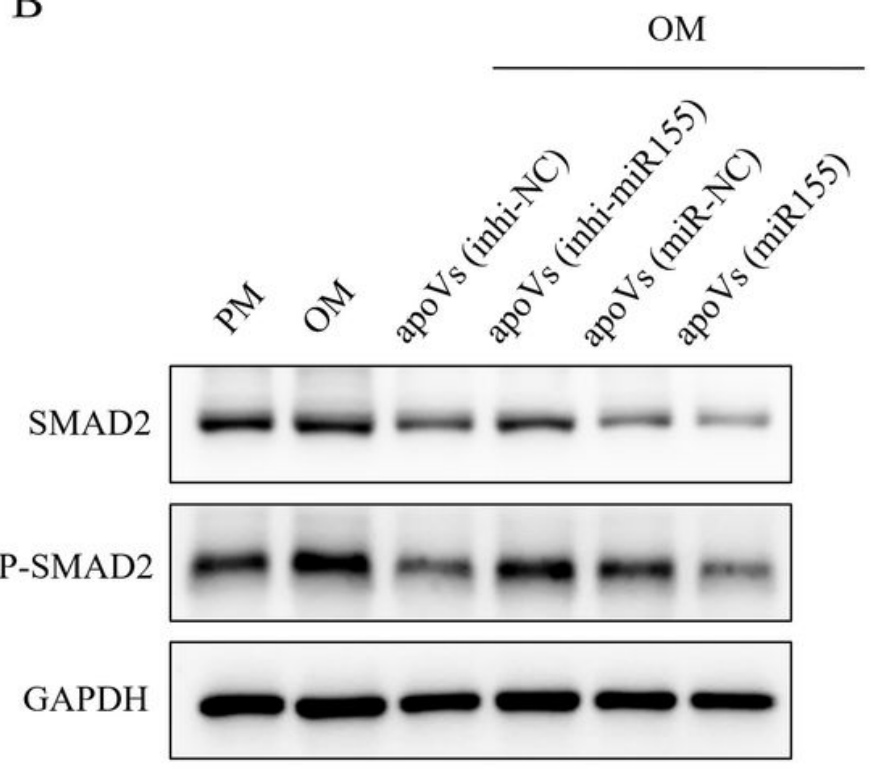

\section{Figure 8}

MiR155 regulated adipogenesis and osteogenesis of MSCs cultured with macrophage-derived apoVs via SMAD2 signaling pathway. A. MiR155 inhibited the expression of SMAD2 and P-SMAD2 during the adipogenic differentiation of MSCs. B. MiR155 inhibited the expression of SMAD2 and P-SMAD2 during the osteogenic differentiation of MSCs.

\section{Supplementary Files}

This is a list of supplementary files associated with this preprint. Click to download.

- FigureS1.tif

- Figures2.tif

- FigureS3.tif 